\title{
Persistent Genital Arousal Disorder: a Biopsychosocial Framework
}

\author{
Robyn A. Jackowich ${ }^{1} \cdot$ Caroline F. Pukall $^{1}$
}

Published online: 6 August 2020

(C) The Author(s) 2020

\begin{abstract}
Purpose of Review Persistent genital arousal disorder (also referred to as genitopelvic dysesthesia or PGAD/GD) is a distressing and largely underrecognized condition characterized by persistent, unwanted genital arousal (sensations, sensitivity, vasocongestion) in the absence of subjective/cognitive arousal and sexual desire. The purpose of this review is to summarize recent findings on biological and psychosocial factors in PGAD/GD as they pertain to the assessment and treatment of this condition. These findings will be considered within a biopsychosocial framework, for the purposes of considering next steps for clinical and research efforts.

Recent Findings A small number of studies have recently examined potential biological aetiologies for PGAD/GD: pharmacological agents, spinal pathology and peripheral nerve involvement. Recent studies have also found that PGAD/GD is associated with a significant negative impact on psychosocial wellbeing and daily functioning as compared to symptom-free individuals. In addition, these results highlight cognitive/affective responses to symptoms (e.g. catastrophizing of symptoms) that may influence outcomes. However, biological and psychological research are rarely integrated in these studies, despite the interrelationship between these factors.

Summary Although PGAD/GD was first described in the scientific literature almost two decades ago, most research on $\mathrm{PGAD} / \mathrm{GD}$ is presented in the form of case studies. Prospective treatment trials that integrate biopsychosocial factors are needed in order to provide effective and efficient care to this population. This research would be facilitated by the development of a patient-reported outcome measure, as well as greater education/awareness among healthcare providers and the public about this distressing condition.
\end{abstract}

Keywords Persistent genital arousal disorder · Genitopelvic dysesthesia $\cdot$ PGAD/GD $\cdot$ Biopsychosocial $\cdot$ Catastrophizing $\cdot$ Fear avoidance

\section{Introduction}

First described by Sandra Leiblum and Sharon Nathan in 2001, persistent genital arousal disorder (or PGAD) is a highly distressing condition, characterized by genital arousal (e.g. genital sensations, sensitivity and/or vasocongestion) that occurs in the absence of subjective/cognitive arousal or sexual desire [1]. For some individuals, a 'flare' of PGAD symptoms

Topical Collection on Female Sexual Dysfunction and Disorder

Robyn A. Jackowich

robyn.jackowich@queensu.ca

Caroline F. Pukall

caroline.pukall@queensu.ca

1 Department of Psychology, Queen's University, 62 Arch Street, Kingston, ON K7L 3N6, Canada may last for hours to days, while for others, these symptoms may be continuously present [2]. Symptoms may be triggered by sexual cues, non-sexual cues or there may be no identifiable trigger [2]. A subset of individuals with PGAD also report experiencing distressing and unwanted spontaneous orgasms [2]. PGAD symptoms are not relieved by any behaviour (e.g. masturbation to orgasm) or over-the-counter remedy $[2,3]$. The symptoms are described as distressing, unwanted and sometimes painful $[2,4,5]$ and are associated with a significant negative impact on psychosocial and daily functioning $[6,7 \bullet \bullet]$.

Given the similarities between PGAD and other forms of genitopelvic discomfort (e.g. vulvodynia: chronic, idiopathic vulvar pain [8]), it has been suggested that PGAD may be best considered a type of 'genitopelvic dysesthesia' (defined as an abnormal, unpleasant sensation) where the primarily unwanted sensation is arousal $[6,8,9 \bullet]$. This shift in conceptualization may help decrease stigma associated with the condition 
(i.e. that genital arousal is always wanted or pleasurable [9•]), and it represents a more accurate description of PGADspecifically that PGAD is a disorder of unpleasant sensations, not high/unwanted sexual desire or high subjective/cognitive arousal. As such, we will refer to the condition as PGAD/genitopelvic dysesthesia (PGAD/GD). More recent and detailed diagnostic criteria for PGAD/GD, based on expert opinion, were proposed by ISSWSH in 2016 [10] and are summarized alongside other proposed diagnostic criteria in Table 1.

There is limited information available about the prevalence of PGAD/GD. One study found $1 \%$ of women attending a sexual health clinic in the UK reported all the Leiblum and Nathan (2001) criteria for PGAD/GD, while 33.3\% reported at least one of the criteria [13]. Another study surveying the first-year undergraduate cohort in psychology at a Canadian university $(N=1641)$ also found approximately $1 \%$ of male and female students reported experiencing all of the Leiblum and Nathan (2001) PGAD/GD criteria at a moderate frequency or higher [14]. PGAD/GD affects individuals of all ages
[2], with $10 \%$ reporting lifelong symptoms. While research has primarily focused on women, there are a growing number of case studies of men who experience similar symptoms [15-20].

Despite approaching two decades since PGAD/GD was first described in the scientific literature, there remains limited research on, and awareness of, PGAD/GD. Research has primarily been in the form of individual case studies (for a recent review, see [3]), and no systematic treatment outcome studies (i.e. controlled trials) have been published. PGAD/GD was only recently listed in a diagnostic manual - the 11th edition of the International Classification of Diseases [21]. This lack of awareness and knowledge of PGAD/GD results in difficulty accessing treatment for these highly distressing symptoms. Indeed, individuals with $\mathrm{PGAD} / \mathrm{GD}$ frequently attribute a lack of healthcare provider knowledge of $\mathrm{PGAD} / \mathrm{GD}$ to be a barrier to receiving timely and effective care [22]. Overall, there is need for more research on this distressing condition as well as a theoretical framework to guide and integrate research and clinical efforts in the biological and psychosocial domains.

Table 1 Proposed diagnostic criteria for persistent genital arousal disorder/genitopelvic dysesthesia (PGAD/GD). Common features of all criteria are noted in italics

\begin{tabular}{|c|c|}
\hline Citation & Proposed PGAD/GD diagnostic criteria \\
\hline $\begin{array}{l}\text { Leiblum and Nathan } \\
\text { (2001) [1] }\end{array}$ & $\begin{array}{l}\text { 1. Physiological responses characteristic of sexual arousal (genital and breast vasocongestion and sensitivity) persist for an } \\
\text { extended period of time (hours to days) and do not subside completely on their own. } \\
\text { 2. The signs of physiological arousal do not resolve with ordinary orgasmic experience and may require multiple orgasms } \\
\text { over hours or days to remit. } \\
\text { 3. These physiological signs of arousal are usually experienced as unrelated to any subjective sense of sexual excitement or } \\
\text { desire. } \\
\text { 4. The persistent sexual arousal may be triggered not only by a sexual activity but also by seemingly non-sexual stimuli or by } \\
\text { no apparent stimulus at all. } \\
\text { 5. The physiological signs of persistent arousal are experienced as unbidden, intrusive and unwanted. When feelings of } \\
\text { genital arousal persist for days, week or even months, they are experienced as personally distressing and worrisome. }\end{array}$ \\
\hline Basson et al. (2004) [11] & $\begin{array}{l}\text { Spontaneous intrusive and unwanted genital arousal (e.g. tingling, throbbing, pulsating) in the absence of sexual interest } \\
\text { and desire. Any awareness of subjective arousal is typically but not in variably unpleasant. The arousal is unrelieved by } \\
\text { one or more orgasms and the feelings of arousal persist for hours or days. }\end{array}$ \\
\hline Parish et al. (2016) [10] & $\begin{array}{l}\text { 1. Characterized by persistent or recurrent, unwanted or intrusive, distressing feelings of genital arousal or being on the } \\
\text { verge of orgasm (genital dysesthesia), not associated with concomitant sexual interest, thoughts or fantasies for }>\text { or equal } \\
\text { to } 6 \text { months. Could be associated with: } \\
\text { 2. Limited resolution, no resolution or aggravation of symptoms by sexual activity with or without aversive and/or } \\
\text { compromised orgasm } \\
\text { 3. Aggravation of genital symptoms by certain circumstances } \\
\text { 4. Despair, emotional lability, catastrophizing and/or suicidality } \\
\text { 5. Inconsistent evidence of genital arousal during symptoms }\end{array}$ \\
\hline $\begin{array}{l}\text { Waldinger et al. }(2018)^{\mathrm{a}} \\
\quad[12]\end{array}$ & $\begin{array}{l}\text { 1. A state of unwanted restless genital sensations that are associated with } \\
\text { 2. restless legs, and/or } \\
\text { 3. complaints of overactive bladder, and/or } \\
\text { 4. urethral hypersensitivity, } \\
\text { 5. and not resolving by sexual activity }\end{array}$ \\
\hline
\end{tabular}

$P G A D / G D$ persistent genital arousal disorder/genitopelvic dysesthesia

${ }^{a}$ These criteria are based on the most recent publication by Waldinger and Schweitzer (2018). Previous publications from Waldinger and colleagues include a different set of diagnostic criteria: the combination of PGAD (defined by Leiblum \& Nathan, 2001) plus the presence of restless leg syndrome, bladder syndrome and/or urethral hypersensitivity 


\section{Biological Factors in PGAD}

Research on biological factors associated with PGAD/GD is beginning to increase, but overall remains relatively limited. This paucity of research leaves healthcare providers who treat PGAD/GD in a position where they must carefully consider and balance the lack of empirically informed treatments and the invasiveness or cost of treatments to their patients/clients.

Multiple aetiologies have been hypothesised for the symptoms of PGAD/GD including vascular factors, central and peripheral nervous system factors, pharmacological factors, dietary and psychosocial factors (for a recent summary of case studies examining causes and treatments of PGAD/GD, see [3]) - suggesting that the aetiology of PGAD/GD may be multifactorial. In addition, like a diagnosis of vulvodynia, some cases of PGAD/GD may be idiopathic. Recent research on biological factors in PGAD/GD has focused on pharmacological causes and interventions, peripheral sensory neuropathy and spinal pathology (e.g. cauda equina syndrome, Tarlov cysts). Each will be briefly summarized here.

\section{Pharmacological Causes and Interventions}

A small number of case reports and self-report studies have observed that pharmacological agents (either initiation or withdrawal) can induce PGAD/GD symptoms - primarily seen with antidepressant medications $[2,23]$. To date, no randomised placebo-controlled clinical trials have been undertaken to investigate pharmacological treatments for PGAD/GD. Current knowledge of these treatments relies exclusively on case reports. Kruger and colleagues provide a recent review of different drug classes (antiandrogens, antidepressants, anticonvulsants, partial agonist at nicotinic acetylcholine receptor subtypes for smoking cessation, dopamine agonists, dopamine antagonists, opioids, toxins and cannabinoids) that have been used to treat PGAD/GD symptoms and the results of the corresponding case reports [23]. Only a small number of cases have examined each medication (i.e. less than 10 cases per medication), with most finding some improvement in PGAD/GD symptoms (improvement found in 25 of 30 cases summarized; [23]). However, Kruger and colleagues note that there is likely an underreporting of treatment failures in the current case report format literature, which would also provide important information about the effectiveness of pharmacological treatments for PGAD/GD [23].

\section{Spinal Pathology}

Komisaruk and Goldstein recently reviewed two proposed aetiologies for PGAD/GD resulting from spinal pathology: Tarlov cysts (i.e. fluid-filled sacs at the spinal nerve root) and cauda equina syndrome [24]. One study found that $66.7 \%(n=12 / 18)$ of spinal MRIs collected from individuals in an online PGAD/GD support group contained a sacral Tarlov cyst [25]. Subsequently, Feigenbaum and Boone found that removal of Tarlov cysts in individuals with PGAD/GD symptoms resulted in improvement in 10/11 women [26]. More recently, a retrospective chart review found that 4 of 10 women presenting with PGAD/GD symptoms at a neurology clinic had Tarlov cysts [27]. Komisaruk and Goldstein also suggest that irritation (via a herniated intervertebral disc) of the roots of the genital sensory nerves within the cauda equina, called cauda equina syndrome, may lead to the development of PGAD/GD symptoms [24] which has been examined in a series of case studies as well [28]. Although these initial studies suggest spinal pathology may be one potential aetiology in PGAD/GD, we still require systematic studies that include measurement of symptomology (i.e. intensity, duration, quality of sensations, associated distress) and functioning (i.e. psychosocial, sexual and relationship wellbeing) following treatment and at long-term follow-up.

\section{Small-Fibre Sensory Neuropathy}

Waldinger and colleagues have hypothesized that small-fibre sensory neuropathy of the pudendal nerve could lead to the dysesthesias that characterize PGAD/GD [12]. In addition to compression at the nerve root ( [24], see above), PGAD/GD symptoms may result from compression or entrapment of more distal areas of the pudendal nerve, including the dorsal nerve of the clitoris [29]. Waldinger and colleagues named PGAD/GD 'restless genital syndrome' when it occurs with a common constellation of comorbidities, specifically restless leg syndrome, overactive bladder syndrome and urethral hypersensitivity [12]. While these authors recommend pelvic and/or lumbosacral MRIs to investigate for spinal or peripheral nerve involvement in PGAD/GD, so far, surgical interventions have only been evaluated in small samples, and larger prospective clinical trials are needed [29, 30].

\section{Psychosocial Factors in PGAD/GD}

A small number of recent studies have examined the psychosocial wellbeing of individuals with $\mathrm{PGAD} / \mathrm{GD}$ using validated self-report measures and control group comparisons. By definition, $\mathrm{PGAD} / \mathrm{GD}$ is associated with distress - however, psychosocial factors may potentially increase one's risk of developing PGAD/GD, mediate PGAD/GD symptoms or may be negatively impacted by $\mathrm{PGAD} / \mathrm{GD}$. 


\section{Psychosocial Risk Factors Associated With PGAD/GD Symptoms and Distress}

Although psychosocial factors alone are unlikely to cause PGAD/GD, they may contribute to the development and maintenance of the disorder and its associated distress. Research in this area is minimal, and studies are limited by their cross-sectional designs. Longitudinal and/or intervention studies are needed to assess the relationships among psychosocial factors, PGAD/GD symptoms and PGAD-related distress. However, the associations described below may serve as a starting point for our understanding of the ways in which psychosocial factors contribute to PGAD/GD symptoms.

Cognitive/Affective Factors In 2007, Leiblum and Chivers proposed a psychological model to explain the development of PGAD/GD symptoms [31•]. They proposed that negative appraisals of spontaneous genital arousal, which is a normative part of female sexuality, may lead to increased anxiety and sympathetic nervous system activity. This in turn could increase genital arousal sensitization and narrow one's attention to these sensations, thus creating a feedback cycle of increased genital arousal sensations. Supporting this model, other studies have found high rates of pre-existing difficulties with mood, anxiety and stress $[7 \cdot \bullet, 31 \cdot]$ in women with PGAD/GD symptoms. Indeed, many women with PGAD/GD self-report that stress (33.98\%), anxiety $(29.13 \%)$ and loss $(13.59 \%)$ were the initial triggers of their symptoms [32]. Jackowich and colleagues found catastrophizing of vulvar sensations (rumination about symptoms, magnification of symptoms, hopelessness) was significantly associated with depression, anxiety, sexual distress, symptom distress and symptom severity $[7 \bullet \bullet]$.

These results highlight the relationship between biological and psychological factors. Cognitive and affective reactions toward symptoms (e.g. hypervigilance, catastrophizing) may mediate the relationship between PGAD/GD symptoms and functional outcomes (e.g. symptom severity, distress, daily functioning). In addition, these cognitive and affective factors may be potential targets of treatment. Facelle and colleagues have recommended mindfulness-based treatments for PGAD/GD symptoms, and one case study by Hiller and Hekster found a couples-based cognitive-behavioural therapy effective in reducing symptoms and improving the overall relationship $[33,34]$.

Personality Factors Certain personality characteristics have also been found to be associated with greater PGAD/GD symptom distress. Carvalho and colleagues found that neuroticism, low openness and sexual conservatism significantly predicted distress associated with PGAD/GD symptoms [35]. Additionally, sexual conservatism moderated the relationship between symptom severity and distress, such that women with higher PGAD symptom severity and higher sexual conservatism reported significantly greater distress then women with higher PGAD symptom severity and lower sexual conservatism [35]. Of note, more research is needed to understand the directionality among these findings, as this was a single-timepoint study.

Sexual Abuse Two studies have inquired about sexual abuse histories in samples of women with PGAD/GD. Both found high rates of childhood sexual abuse $(46.7-52.6 \%$; $[4,36])$. Direct comparisons are challenging due to differences in the wording of questions about past sexual abuse; however, the rates of childhood sexual abuse reported by individuals with PGAD/GD appear to be higher than those reported by individuals with other forms of genitopelvic discomfort (e.g. vulvodynia, chronic pelvic pain [37-39]). Although more research is needed in larger samples of individuals with PGAD/GD, sexual abuse experiences may negatively influence evaluations of arousal and/or emotional responses to normative or dysfunctional spontaneous genital arousal. Indeed, Carvalho and colleagues found that individuals with PGAD/GD report more negative thoughts, increased negative affect and decreased positive affect during sexual activity than women without PGAD/GD, which could result from a history of abuse and/or from the PGAD/GD symptoms themselves [40]. However, the association between sexual abuse and PGAD, as well as the timing of these associations, has yet to be investigated. A history of sexual and physical abuse is a risk factor for other forms of genitopelvic discomfort, such as vulvodynia [39] and other forms of chronic pelvic pain [41].

\section{Psychosocial Consequences of PGAD/GD}

Depression, Anxiety and Stress Three recent studies have noted that individuals with PGAD/GD report significantly greater mental health concerns than individuals without these symptoms [7••, 40, 42]. Compared to individuals without PGAD/GD, an online sample of women with PGAD/GD reported significantly poorer psychosocial wellbeing on all subscales of the Brief Symptom Inventory scale, except interpersonal sensitivity [40]. Another study found that among women with PGAD/GD, average scores on measures of depression, anxiety and stress fell above clinical cut-offs [42]. A third study found that women with PGAD/GD symptoms reported significantly greater depression and anxiety symptoms than age-matched women without PGAD/GD symptoms [7••]. Over half $(54 \%)$ of women reported some degree of suicidal ideation - much higher rates than are seen in the general population (e.g. from 2.3 to $14.6 \%$ across 5 countries; [43]) and in those with chronic pain conditions (prevalence rate of approximately 20\% [44]). It is important to note that this research is primarily cross- 
sectional; therefore, we cannot determine the direction of causality. These findings highlight the importance of a thorough clinical assessment of psychosocial wellbeing with individuals experiencing PGAD/GD, so that appropriate support can be provided.

Daily Functioning Likely contributing to the psychosocial difficulties associated with PGAD/GD, research has found PGAD/GD interferes with many activities of daily living, including social activities and work [6]. PGAD/GD symptoms that are described as 'painful' additionally interfere with intermediate activities of daily living (e.g. driving, housework). In an online sample of women with PGAD/GD symptoms, the most commonly reported activities affected by PGAD/GD symptoms included sitting (85.2\%), concentrating (83.5\%), social activities $(69.6 \%)$, work $(67.8 \%)$, wearing tight fitting clothing $(67.8 \%)$ and sleep $(67.8 \%$; [2]).

Sexual and Relationship Functioning Like other forms of genitopelvic discomfort (e.g. vulvodynia, [39]), PGAD/GD also appears to have negative consequences for sexuality more broadly. Women with PGAD/GD report fewer erotic thoughts, greater negative affect and less positive affect during sexual activity [40]. Squibb and colleagues found an association between greater numbers of other medical conditions, greater depression, greater anxiety and higher levels of sexual distress in women with $\mathrm{PGAD} / \mathrm{GD}$ [42]. Women with PGAD/GD report poorer sexual functioning than women without PGAD/GD on all domains of the Female Sexual Function Index, except desire [45]. Many women with PGAD/GD also report avoiding sexual activity in order to reduce symptoms $[7 \bullet \bullet]$. This is reflected in the high levels of sexual distress seen in individuals with $\mathrm{PGAD} / \mathrm{GD}$ across multiple studies [7••, 42]. Little is known about the impact that PGAD/GD has on relationships, though women with PGAD/GD reported significantly lower relationship satisfaction than age-matched women without PGAD/GD symptoms $[7 \bullet \bullet]$.

\section{Integrating Mind and Body: a Biopsychosocial Framework}

Most of the research to date on PGAD/GD has focused on possible biological underpinnings of the condition and psychosocial outcomes of having the condition. The predominant literature on intervention has focused on medication and physiological treatments, ranging from transcutaneous electrical nerve stimulation to surgery (e.g. removal of Tarlov cysts; [3]). Very little is known about long-term treatment outcomes and what options are, in fact, effective even in the short term. In addition, many cases of PGAD/GD have unknown aetiology. A potential downfall of the early literature in terms of the conceptualization of and treatment approaches to PGAD/GD is the assumption that PGAD/GD is a purely biological condition that can only be treated by medical/surgical means. Though this biomedical perspective may be supported by the existing literature, it is important to stress that the symptoms of PGAD/GD will have an impact on psychosocial functioning. The psychosocial experience can, in turn, influence the experience and maintenance of the symptoms; therefore, psychosocial factors can also be targeted for potential symptom management.

The recognition of the shared importance of the biological and psychosocial components of a condition is well represented by the biopsychosocial perspective, a model often used in the chronic pain literature [46]. This model has been applied to vulvodynia [47], which has some shared characteristics with PGAD/GD [8]; for example, their common comorbidities and features are consistent with central sensitization. As described above and consistent with this view, Pukall and colleagues proposed that all forms of genitopelvic discomfort, including vulvodynia and PGAD/GD, be conceptualized as genitopelvic dysesthesias [9॰]. They further suggested that each form of genitopelvic dysesthesia be classified by cause of symptoms (known or idiopathic), much like the most recent classification of chronic vulvar pain and vulvodynia [47]. Importantly, this approach allows for the future incorporation of 'potential associated factors' (e.g. pelvic floor musculature, psychosocial factors) - which are biopsychosocial in nature - to idiopathic GP/PGAD, in the same manner as they have been applied to vulvodynia [47]. Although the research on $\mathrm{PGAD} / \mathrm{GD}$ is not yet sufficiently broad or well developed to delineate what biopsychosocial factors may be potentially associated with PGAD/GD, it is essential to adopt, at the very least, a biopsychosocial perspective of PGAD/GD as early as possible in order to prevent dualistic theoretical and practical approaches to $\mathrm{PGAD} / \mathrm{GD}$ treatment.

A biopsychosocial approach to $\mathrm{PGAD} / \mathrm{GD}$ is supported by two main lines of evidence. First, one's interpretation of the sensations of genital arousal can influence one's distress level. For example, as suggested by Leiblum and Chivers (2007), a negative appraisal of arousal sensations may lead to distress associated with these sensations, whereas a positive interpretation may result in no distress to, or even a positive experience of, the sensations [31•]. Indeed, there is evidence of individuals who have persistent sensations of genital arousal but who are not distressed by the sensations (e.g. [13]), suggesting that one's reaction to the sensations (as determined by psychosocial influences, for example) can play a significant role in the distress experienced. The continuum of possible reactions to the symptoms may be primarily influenced by psychosocial factors, thereby supporting the importance of an approach that accounts for psychosocial factors. Related to this point is the emerging evidence (summarized above) of cognitive and emotional mediators of symptoms and distress (e.g. catastrophizing, 
hypervigilance), which again suggest that psychosocial factors play a role in the experience of the symptoms and may be potential targets for treatment (e.g. cognitive skills to decrease catastrophizing and hypervigilance). Second, the significant psychosocial impact of PGAD/GD symptoms on people's lives (e.g. work, social activities), as demonstrated by several studies (e.g. $[6,40])$, cannot be ignored and will likely require attention over and above symptom reduction. Approaches to PGAD/GD that incorporate all elements of the biopsychosocial model will therefore allow for a more comprehensive conceptualization of PGAD/GD, which will then likely lead to a multidisciplinary assessment and treatment approach. The fear-avoidance model (FA model; $[48,49]$ ) is one such approach that can prove useful in $\mathrm{PGAD} / \mathrm{GD}$.

\section{Application of the Fear-Avoidance Model to PGAD/GD}

An example of a model integrating both biological and psychological factors in our understanding of chronic pain is the fear-avoidance model (FA model; [48, 49]). This model may also help guide future research and the development of clinical interventions for PGAD/GD. The FA model of chronic pain specifies that the way pain is interpreted may lead to different outcomes. If individuals interpret pain as non-threatening and maintain their activities, then recovery from pain is promoted. Alternatively, if one is hypervigilant to pain, catastrophizes, fears and avoids pain, what may initially be adaptive in the acute phase of pain may paradoxically lead to poorer outcomes and the development of chronic pain [48, 49]. The FA model has been previously applied to other forms of genital discomfort (e.g. vulvodynia, [50]) which, as described above, share similarities with PGAD/GD.

While the predictive validity of the FA model in PGAD/GD has yet to be tested, research supports the discrete variables within the model. Figure 1 presents the FA model, modified for PGAD/GD. Evidence that supports each part of the model is cited beneath. For example, previous research has found high rates of PGAD/GD catastrophizing (e.g. 'I become afraid that the sensations will get worse') were positively associated with symptom severity, distress and symptoms of depression and anxiety [7••]. Application of the FA model to PGAD/GD would provide information about psychosocial factors that contribute to the development and maintenance of this condition.

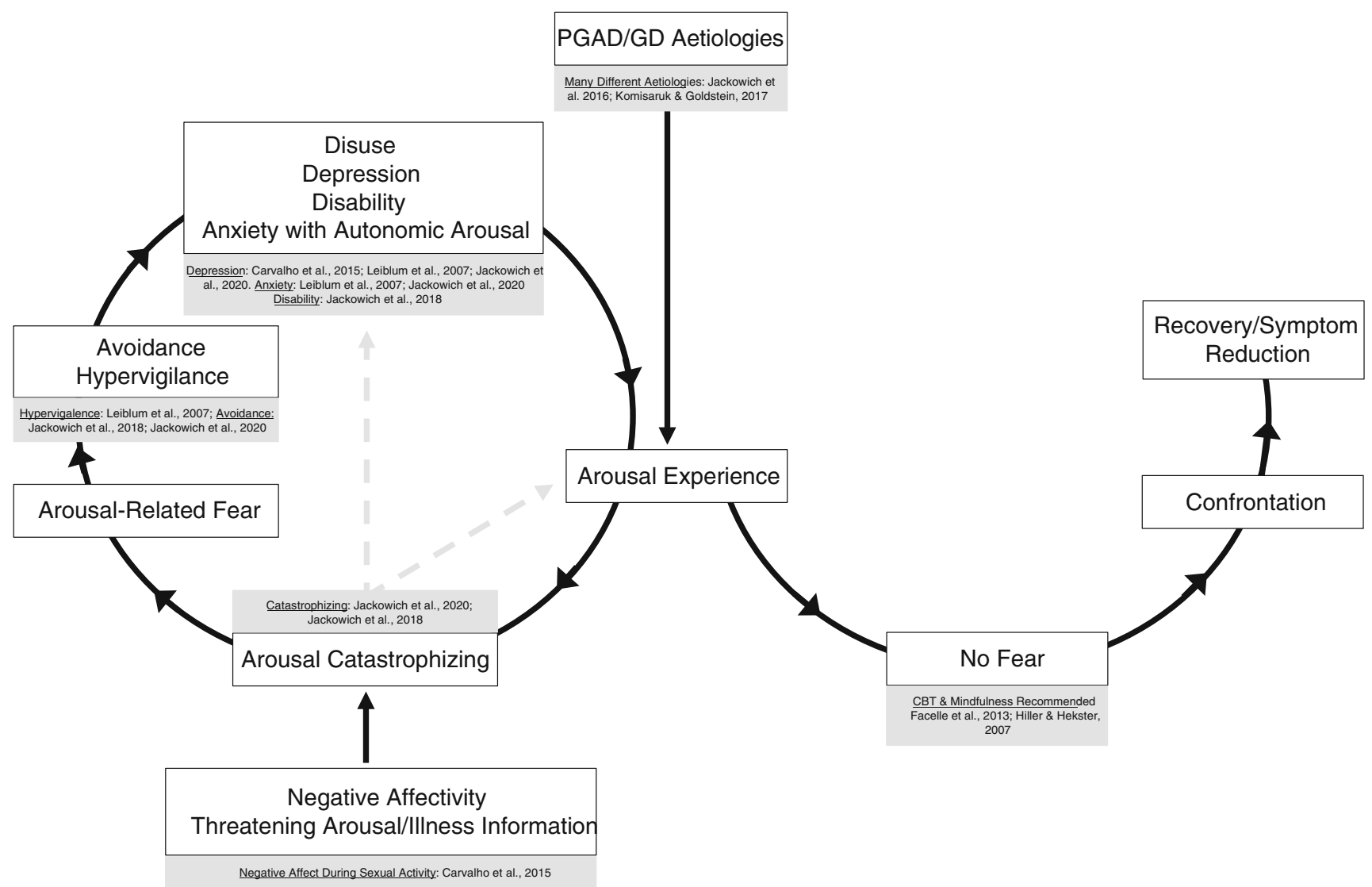

Fig. 1 The fear-avoidance model is presented here based on Vlaeyen and Linton (2000) [49] and modified theoretically for persistent genital arousal disorder/genitopelvic dysesthesia (PGAD/GD) $[2-8,9 \bullet]$. The model is presented in white boxes with circular black arrows, while citations for past studies that have examined factors related to this model are presented in grey. Dotted lines represent findings that have found associations between variables within the model (e.g. association between catastrophizing and greater depression and anxiety symptoms: [6]) 


\section{Conclusions: Future Research and Clinical Considerations}

Research on PGAD/GD is beginning to emerge, but it is still in its infancy. To date, studies have examined biological and psychosocial factors separately, failing to investigate or acknowledge the interrelationship between these components. In addition, the majority of research on PGAD is in the form of case studies, which are limited in their ability to assess the effectiveness of treatments for PGAD. A literature predominantly consisting of case studies may also underrepresent studies in which symptom improvement was not found. The chronic pain literature, which is more established, may provide a framework for guiding future research and clinical efforts from a biopsychosocial perspective. Longitudinal and/or experimental studies are needed to better understand and evaluate biopsychosocial models of PGAD/GD - such as the fear-avoidance model. Recognizing the ways in which biopsychosocial factors influence one another may highlight potential areas for intervention and support.

Clinical assessment of PGAD/GD should include careful consideration of contributing psychosocial factors and resulting psychosocial impairment. For example, individuals with PGAD/GD should be carefully screened for suicidal ideation given the high rates found in recent studies $[7 \bullet \cdot]$ and these individuals should be connected with support as needed.

Current recommendations for interventions are multidisciplinary, including pharmacotherapy, physiotherapy of the pelvic floor and psychotherapy [23, 51]. Given the limited empirical support for all treatment interventions, healthcare providers treating individuals with PGAD/GD must balance this limited empirical evidence with the invasive or costly nature of proposed treatments.

Given the highly distressing nature of $\mathrm{PGAD} / \mathrm{GD}$, there is great need for clinical trials evaluating the effectiveness of different medical, physiotherapy and psychosocial interventions. The development of a patient-reported outcome instrument could better assess treatment outcomes and quality of life. As a starting point, we may look to the Initiative on Methods, Measurement and Pain Assessment in Clinical Trials (IMMPACT) guidelines, as has been done for vulvodynia [52•]. Similar to the IMMPACT recommendations for vulvodynia, the inclusion of measures that target daily functioning, sexuality, psychosocial and relationship wellbeing would also be important for assessing outcomes in PGAD/GD. Such trials would be strengthened by including measures of biopsychosocial outcomes/psychosocial factors. Psychosocial factors themselves (e.g. catastrophizing) may also be a target of intervention.

Finally, the lack of public and healthcare knowledge of PGAD/GD serves as a barrier to both conducting research and accessing care for these symptoms. Knowledge translation efforts about PGAD/GD are an important step. Indeed, sexual arousal is often interpreted as an exclusively positive and wanted experience [9॰]. We may look to efforts in other domains of sexuality, and even genitopelvic pain such as the \#Itsnotinyourhead campaign. Some initial efforts have been made in this regard (for example, the \#PGADFacts campaign from our team at the Queen's University Sexual Health Research Lab. See https://sexlab.ca/pgad for more information). Continued efforts to increase awareness of PGAD/GD in the healthcare community and more broadly may facilitate increased awareness of this distressing condition.

\section{Compliance with Ethical Standards}

Conflict of Interest Dr. Pukall reports grants from CIHR, grants from ISSWSH, and grants from Queen's University, during the conduct of the study.

Robyn Jackowich reports grants from ISSWSH, outside the submitted work.

Human and Animal Rights and Informed Consent This article does not contain any studies with human or animal subjects performed by any of the authors.

Open Access This article is licensed under a Creative Commons Attribution 4.0 International License, which permits use, sharing, adaptation, distribution and reproduction in any medium or format, as long as you give appropriate credit to the original author(s) and the source, provide a link to the Creative Commons licence, and indicate if changes were made. The images or other third party material in this article are included in the article's Creative Commons licence, unless indicated otherwise in a credit line to the material. If material is not included in the article's Creative Commons licence and your intended use is not permitted by statutory regulation or exceeds the permitted use, you will need to obtain permission directly from the copyright holder. To view a copy of this licence, visit http://creativecommons.org/licenses/by/4.0/.

\section{References}

Papers of particular interest, published recently, have been highlighted as:

- Of importance

•- Of major importance

1. Leiblum SR, Nathan SG. Persistent sexual arousal syndrome: a newly discovered pattern of female sexuality. J Sex Marital Ther. 2001;27(4):365-80.

2. Jackowich R, Pink L, Gordon A, Poirier É, Pukall CF. Symptom characteristics and medical history of an online sample of women who experience symptoms of persistent genital arousal. J Sex Marital Ther. 2018;44(2):111-26.

3. Jackowich RA, Pink L, Gordon A, Pukall CF. Persistent genital arousal disorder: a review of its conceptualizations, potential origins, impact, and treatment. Sex Med Rev. 2016;4(4):329-42.

4. Pink L, Rancourt V, Gordon A. Persistent genital arousal in women with pelvic and genital pain. J Obstet Gynaecol Can. 2014;36(4): 324-30.

5. Waldinger MD, Van Gils APG, Ottervanger HP, Vandenbroucke WVA, Tavy DLJ. Persistent genital arousal disorder in 18 Dutch 
women: part I. MRI, EEG, and transvaginal ultrasonography investigations. J Sex Med. 2009;6(2):474-81.

6. Jackowich RA, Pink L, Gordon A, Poirier É, Pukall CF. An online cross-sectional comparison of women with symptoms of persistent genital arousal, painful persistent genital arousal, and chronic vulvar pain. J Sex Med. 2018;15(4):558-67.

7.• Jackowich RA, Poirier É, Pukall CF. A comparison of medical comorbidities, psychosocial, and sexual well-being in an online cross-sectional sample of women experiencing persistent genital arousal symptoms and a control group. J Sex Med. 2020;17(1): 69-82. A recent article, utilizing an age-matched control group, highlighting the high rates of depression, anxiety, and sucidial ideation in women with GD/PGAD as well as the association of greater catastrophizing (of vulvar sensations), and poorer symptom, and psychosocial outcomes.

8. Markos AR, Dinsmore W. Persistent genital arousal and restless genitalia: sexual dysfunction or subtype of vulvodynia? Int J STD AIDS. 2013;24(11):852-8.

9. Pukall CF, Jackowich R, Mooney K, Chamberlain SM. Genital sensations in persistent genital arousal disorder: a case for an overarching nosology of genitopelvic dysesthesias? Sex Med Rev. 2019;7(1):2-12 .A recent review highlighting the similarities between GD/PGAD and other forms of genitopelvic discomfort. This article also makes a case for new terminology and classification of unwanted genitopelvic sensations: genitopelvic dysesthesias.

10. Parish SJ, Goldstein AT, Goldstein SW, Goldstein I, Pfaus J, Clayton AH, et al. Toward a more evidence-based nosology and nomenclature for female sexual dysfunctions - part II. J Sex Med. 2016;13(12):1888-906.

11. Basson R, Leiblum S, Brotto L, Derogatis L, Fourcroy J, FuglMeyer K, et al. Revised definitions of women's sexual dysfunction. J Sex Med. 2004;1(1):40-8

12. Waldinger MD, Schweitzer DH. Restless genital syndrome (ReGS) should be distinguished from spontaneous orgasms: a case report of cannabis-induced spontaneous orgasm. J Sex Marital Ther. 2018;44(3):231-5.

13. Garvey LJ, West C, Latch N, Leiblum S, Goldmeier D. Report of spontaneous and persistent genital arousal in women attending a sexual health clinic. Int J STD AIDS. 2009;20:519-21.

14. Jackowich RA, Pukall CF. Prevalence of persistent genital arousal disorder symptoms in a cross-sectional sample of Canadian undergraduate students. J Sex Med. 2017;14(6):e368-9.

15. Dikici S, Ince Gunal D, Arslan G, Ali Kayıkc1 M, Suber DA. Restless genital syndrome in a male patient relieved by pramipaxol and gabapentin. Neurol Asia. 2015;20(4):405-6.

16. Serefoglu EC. Restless genital syndrome in a 38-year-old man: a case report. J Sex Med. 2016;13(5):S210.

17. Ahmad I, Rashid S, Rathore FA. Restless genital syndrome: case report of a rare disorder from Pakistan. Cureus. 2018;10(5):e2619.

18. Waldinger MD, Venema PL, Van Gils APG, De Lint GJ, Schweitzer DH. Stronger evidence for small fiber sensory neuropathy in restless genital syndrome: two case reports in males. J Sex Med. 2011;8(1):325-30.

19. Kamatchi R, Ashley-Smith A. Persistent genital arousal disorder in a male: a case report and analysis of the cause. Br J Med Pract. 2013;6(1):30-2.

20. Goldstein I, Komisaruk BR, Rubin RS, Goldstein SW, Elliott S, Kissee J, et al. A novel collaborative protocol for successful management of penile pain mediated by radiculitis of sacral spinal nerve roots from tarlov cysts. Sex Med. 2017 Sep;5(3):e203-11.

21. World Health Organziation. International classification of diseases for mortality and morbidity statistics. 11th Editi. 2018.

22. Jackowich R, Pink L, Gordon A, Pukall C. 059 health care experiences of women with symptoms of persistent genital arousal. J Sex Med. 2017;14(6):e369.
23. Kruger THC, Schippert C, Meyer B. The pharmacotherapy of persistent genital arousal disorder. Curr Sex Heal Reports. 2020;12(1): $34-9$.

24. Komisaruk BR, Goldstein I. Persistent genital arousal disorder: current conceptualizations and etiologic mechanisms. Curr Sex Heal Reports. 2017;9(4):177-82.

25. Komisaruk BR, Lee H. Prevalence of sacral spinal (Tarlov) cysts in persistent genital arousal disorder. J Sex Med. 2012;9(8):2047-56.

26. Feigenbaum F, Boone K. Persistent genital arousal disorder caused by spinal meningeal cysts in the sacrum: successful neurosurgical treatment. Obstet Gynecol. 2015;126(4):839-43.

27. Oaklander AL, Sharma S, Kessler K, Price BH. Persistent genital arousal disorder: a special sense neuropathy. Pain Reports. 2020;5(1):e801.

28. Kim C, Blevins J, Hanley J, Goldstein S, Komisaruk B, Goldstein I. 019 neurogenic persistent genital arousal disorder (PGAD) secondary to radiculopathy of sacral spinal nerve roots (SSNR): treatment outcome following spine surgery. J Sex Med. 2019;16(6):S9.

29. Klifto KM, Dellon AL. Persistent genital arousal disorder: review of pertinent peripheral nerves. Sex Med Rev. 2019; Epub ahead of print.

30. Klifto K, Dellon AL. Persistent genital arousal disorder: treatment by neurolysis of dorsal branch of pudendal nerve. Microsurgery. 2019;40(2):160-6.

31. Leiblum SR, Chivers ML. Normal and persistent genital arousal in women: New perspectives. J Sex Marital Ther. 2007;33:357-73. An earlier article that provides a theoretical framework for the role of cognitive and affective reactions to GD/PGAD symtpoms and how this might affect symptom outcomes: integrating both biological and psychosocial factors.

32. Leiblum S, Brown C, Wan J, Rawlinson L. Persistent sexual arousal syndrome: a descriptive study. J Sex Med. 2005;2(3):331-7.

33. Facelle TM, Sadeghi-Nejad H, Goldmeier D. Persistent genital arousal disorder: characterization, etiology, and management. J Sex Med. 2013;10(2):439-50.

34. Hiller J, Hekster B. Couple therapy with cognitive behavioural techniques for persistent sexual arousal syndrome. Sex Relatsh Ther. 2007;22(1):91-6.

35. Carvalho J, Veríssimo A, Nobre PJ. Psychological factors predicting the distress to female persistent genital arousal symptoms. J Sex Marital Ther. 2015;41(1):11-24.

36. Leiblum S, Seehuus M, Goldmeier D, Brown C. Psychological, medical, and pharmacological correlates of persistent genital arousal disorder. J Sex Med. 2007;4(5):1358-66.

37. Jamieson DJ, Steege JF. The association of sexual abuse with pelvic pain complaints in a primary care population. Am J Obstet Gynecol. 1997;177(6):1408-12.

38. Lampe A, Sölder E, Ennemoser A, Schubert C, Rumpold G, Söllner W. Chronic pelvic pain and previous sexual abuse. Obstet Gynecol. 2000;96(6):929-33.

39. Pukall CF, Goldstein AT, Bergeron S, Foster D, Stein A, KelloggSpadt S, et al. Vulvodynia: definition, prevalence, impact, and pathophysiological factors. J Sex Med. 2016;13(3):291-304.

40. Carvalho J, Veríssimo A, Nobre PJ. Cognitive and emotional determinants characterizing women with persistent genital arousal disorder. J Sex Med. 2013;10(6):1549-58.

41. Latthe P, Mignini L, Gray R, Hills R, Khan K. Factors predisposing women to chronic pelvic pain: systematic review. BMJ. 2006;332(7544):749-55.

42. Squibb L, Stepleman L, Goldstein I, Sand M, Zamboni B. Predictors and moderators of sexually related distress in women with persistent genital arousal disorder. Int J Sex Health. 2019;31(4):1-13.

43. Casey PR, Dunn G, Kelly BD, Birkbeck G, Dalgard OS, Lehtinen $\mathrm{V}$, et al. Factors associated with suicidal ideation in the general 
population: five-centre analysis from the ODIN study. Br $\mathrm{J}$ Psychiatry. 2006;189(5):410-5.

44. Tang NKY, Crane C. Suicidality in chronic pain: a review of the prevalence, risk factors and psychological links. Psychol Med. 2006;36(5):575-86.

45. Leiblum SR, Seehuus M. FSFI scores of women with persistent genital arousal disorder compared with published scores of women with female sexual arousal disorder and healthy controls. J Sex Med. 2009;6(2):469-73.

46. Gatchel RJ. Comorbidity of chronic pain and mental health disorders: the biopsychosocial perspective. Am Psychol. 2004;59(8): 795-805.

47. Bornstein J, Goldstein AT, Stockdale CK, Bergeron S, Pukall C, Zolnoun D, et al. 2015 ISSVD, ISSWSH, and IPPS consensus terminology and classification of persistent vulvar pain and vulvodynia. J Sex Med. 2016;13(4):607-12.

48. Leeuw M, Goossens MEJB, Linton SJ, Crombez G, Boersma K, Vlaeyen JWS. The fear-avoidance model of musculoskeletal pain: current state of scientific evidence. J Behav Med. 2007;30(1):7794.
49. Vlaeyen JWS, Linton SJ. Fear-avoidance and its consequences in chronic musculoskeletal pain: a state of the art. Pain. 2000;85(3): 317-32.

50. Thomtén J, Lundahl R, Stigenberg K, Linton S. Fear avoidance and pain catastrophizing among women with sexual pain. Women Health. 2014;10(6):571-81.

51. Pukall CF, Goldmeier D. Persistent genital arousal disorder. In: Binik YM, Hall K, editors. Principles and practice of sex therapy (6th ed). 6th ed. New York, NY: Guilford Press; 2020. p. 488-503.

52. Pukall CF, Bergeron S, Brown C, Bachmann G, Wesselmann U. Recommendations for self-report outcome measures in vulvodynia clinical trials. Clin J Pain. 2017;33(8):756-65. As we look to evaluating different treatment interventions for GD/PGAD, this article may serve as a helpful guide in selecting/developing self-report outcomes for prospective clinical trials.

Publisher's Note Springer Nature remains neutral with regard to jurisdictional claims in published maps and institutional affiliations. 